\title{
Weighted composition operators between Besov-type spaces
}

\author{
Hamid Vaezi*(D), Sima Houdfar (D) \\ Department of Pure Mathematics, Faculty of Mathematical Sciences University of Tabriz, Tabriz, Iran
}

\begin{abstract}
In this paper, we study the boundedness and the compactness of weighted composition operators between Besov-type spaces. Also, we give a Carleson measure characterization of weighted composition operators on Besov spaces.
\end{abstract}

Mathematics Subject Classification (2010). 47B33, 30H25

Keywords. (Weighted) composition operator, Besov-type space, Carleson measure

\section{Introduction}

Let $\mathbb{D}$ be the open unit disc in the complex plane $\mathbb{C}$. Denote by $H(\mathbb{D})$ the class of all complex-valued functions analytic on $\mathbb{D}$. Suppose $\varphi$ and $\psi$ are holomorphic functions defined on $\mathbb{D}$ such that $\varphi(\mathbb{D}) \subseteq \mathbb{D}$. The weighted composition operator $W_{\varphi, \psi}$ induced by $\varphi$ and $\psi$ on $H(\mathbb{D})$ is defined by

$$
W_{\varphi, \psi} f(z)=\psi(z) f(\varphi(z))=\psi(z) C_{\varphi}(f),
$$

for all $f \in H(\mathbb{D})$ and $z \in \mathbb{D}$. When $\psi(z) \equiv 1$, the composition operator $W_{\varphi, 1}$ is denoted by $C_{\varphi}$, i.e.,

$$
W_{\varphi, 1} f(z)=f(\varphi(z))=C_{\varphi}(f),
$$

for all $f \in H(\mathbb{D})$ and $z \in \mathbb{D}$. For the study of composition operators one can refer to [7] and [11].

Fix any $a \in \mathbb{D}$ and let $\sigma_{a}(z)$ be the Mobius transform defined by

$$
\sigma_{a}(z)=\frac{a-z}{1-\bar{a} z}, z \in \mathbb{D} .
$$

We denote the set of all Mobius transformations on $\mathbb{D}$ by $G$. The inverse of $\sigma_{a}$ under composition is again $\sigma_{a}$ for $a \in \mathbb{D}$. Further, we have

$$
\left|\sigma_{a}^{\prime}(z)\right|=\frac{1-|a|^{2}}{|1-\bar{a} z|^{2}}
$$

and

$$
1-\left|\sigma_{a}(z)\right|^{2}=\frac{\left(1-|a|^{2}\right)\left(1-|z|^{2}\right)}{|1-\bar{a} z|^{2}}=\left(1-|z|^{2}\right)\left|\sigma_{a}^{\prime}(z)\right|,
$$

for every $a, z \in \mathbb{D}$.

\footnotetext{
*Corresponding Author.

Email addresses: hvaezi@tabrizu.ac.ir (H. Vaezi), s.hoodfar@tabrizu.ac.ir (S. Houdfar)

Received: 16.10.2017; Accepted: 11.09.2018
} 
For $1 \leq p<\infty, L^{p}(\mathbb{D}, d A)$ will denote the Banach space of Lebesgue measurable functions $f$ on $\mathbb{D}$ with

$$
\|f\|_{p}=\left(\int_{\mathbb{D}}|f(z)|^{p} d A(z)\right)^{\frac{1}{p}}<+\infty,
$$

where $d A(z)$ denote the Lebesgue area measure on $\mathbb{D}$.

For $p=+\infty, L^{\infty}(\mathbb{D}, d A)$ will denote the Banach space of Lebesgue measurable functions $f$ on $\mathbb{D}$ with

$$
\|f\|_{\infty}=e s s \sup \{|f(z)|: z \in \mathbb{D}\}<+\infty .
$$

For $1 \leq p<\infty$, the Bergman space $A^{p}$, is defined to be the subspace of $L^{p}(\mathbb{D}, d A)$ consisting of analytic functions, i.e. $A^{p}(\mathbb{D})=L^{p}(\mathbb{D}) \cap H^{\infty}(\mathbb{D})$. The Bergman spaces are Banach spaces.

For $1 \leqslant p<+\infty$ and $-1<r<+\infty$, the (weighted) Bergman space $A_{r}^{p}=A_{r}^{p}(\mathbb{D})$ of the disc is the space of analytic functions in $L^{p}\left(\mathbb{D}, d A_{r}\right)$, where

$$
d A_{r}(z)=(r+1)\left(1-|z|^{2}\right)^{r} d A(z) .
$$

If $f$ is in $A_{r}^{p}$, we write

$$
\|f\|_{A_{r}^{p}}=\left(\int_{\mathbb{D}}|f(z)|^{p} d A_{r}(z)\right)^{\frac{1}{p}} .
$$

When $1 \leqslant p<+\infty$, the space $A_{r}^{p}$ is a Banach space with the above norm.

For $1<p<+\infty$ and $-1<r<\infty$, an analytic function $f$ on $\mathbb{D}$ is said to belong to the Besov-type space $B_{p, r}$ if

$$
\|f\|_{B_{p, r}}=\left(\int_{\mathbb{D}}\left|f^{\prime}(z)\right|^{p}\left(1-|z|^{2}\right)^{r} d A(z)\right)^{\frac{1}{p}}<\infty,
$$

where $d A(z)$ denote the Lebegue area measure on $\mathbb{D}$. Also, if we take $1<p<\infty$ and $r=p-2$ in (1.3), then we get analytic Besov space, simply denoted by $B_{p}$. We can see that $|f(0)|+\|f\|_{B_{p, r}}$ is a norm on $B_{p, r}$, that makes it a Banach space. Moreover, we can observe that, for $f$ to be in $B_{p, r}$, it is necessary that the derivative of $f$ belong to the weighted Bergman spaces $A_{r}^{p}$.

Definition 1.1. Let $\mu$ be a positive measure on $\mathbb{D}$. Then the space $\mathbb{D}_{p}(\mu)$ is defined as the space of all holomorphic functions $f \in H(\mathbb{D})$ for which $f^{\prime} \in L^{p}(\mathbb{D}, \mu)$. Also, the norm on $\mathbb{D}_{p}(\mu)$ is defined as

$$
\|f\|_{\mathbb{D}_{p}(\mu)}^{p}=\int_{\mathbb{D}}\left|f^{\prime}(z)\right|^{p} d \mu(z) .
$$

Take $0<p<\infty$. A positive measure $\mu$ on $\mathbb{D}$ is called a $p$-Carleson measure in $\mathbb{D}$ if

$$
\sup _{I \subseteq \partial \mathbb{D}} \frac{\mu(S(I))}{|I|^{p}}<\infty
$$

where $|I|$ denotes the arc length of $I$ and $S(I)$ denotes the Carleson square based on $I$,

$$
S(I)=\left\{z \in \mathbb{D}: 1-|I| \leq|z|<1, \frac{z}{|z|} \in I\right\} .
$$

Again, $\mu$ is called a vanishing $p$-Carleson measure if

$$
\lim _{|I| \rightarrow 0} \frac{\mu(S(I))}{|I|^{p}}=0 .
$$

Take $h \in(0,1)$ and $\theta \in[0,2 \pi)$. If we set

$$
S(h, \theta)=\left\{z \in \mathbb{D}:\left|z-e^{i \theta}\right|<h\right\},
$$

then we can see that (1.4) and (1.5) are equivalent to

$$
\sup _{h \in(0,1), \theta \in[0,2 \pi)} \frac{\mu(S(h, \theta))}{h^{p}}<\infty
$$


and

respectively.

$$
\lim _{h \rightarrow 0} \sup _{\theta \in[0,2 \pi)} \frac{\mu(S(h, \theta))}{h^{p}}=0
$$

Suppose $\varphi$ is a holomorphic mapping defined on $\mathbb{D}$. Let $\varphi(\mathbb{D}) \subseteq \mathbb{D}$ and $\psi \in B_{q, r}$ be such that $\psi(z) \varphi^{\prime}(z) \in L^{q}\left(\mathbb{D}, d A_{r}\right)$. We define the measures $\mu_{q, r}$ and $\nu_{q, r}$ on $\mathbb{D}$ by

$$
\mu_{q, r}(E)=\int_{\varphi^{-1}(E)}\left|\psi(z) \varphi^{\prime}(z)\right|^{q}\left(1-|z|^{2}\right)^{r} d A(z)
$$

and

$$
\nu_{q, r}(E)=\int_{\varphi^{-1}(E)}\left|\psi^{\prime}(z)\right|^{q}\left(1-|z|^{2}\right)^{r} d A(z),
$$

where $E$ is a measurable subset of the unit disc $\mathbb{D}$.

If $\psi \in A_{r}^{q}$, then we can define the measure $\nu_{q, \psi, r}$ on $\mathbb{D}$ by

$$
\nu_{q, \psi, r}(E)=\int_{\varphi^{-1}(E)}|\psi(z)|^{q}\left(1-|z|^{2}\right)^{r} d A(z) .
$$

Definition 1.2. Take $1<p<+\infty$ and $-1<r<\infty$. Let $\mu$ be a positive measure on $\mathbb{D}$. Then the measure $\mu$ is $(p, r)$-Carleson measure for $B_{p, r}$ if there is a constant $K>0$ such that

$$
\int_{\mathbb{D}}\left|f^{\prime}(w)\right|^{p} d \mu(w) \leqslant K\|f\|_{B_{p, r}}^{p},
$$

for all $f \in B_{p, r}$. That is, the inclusion operator $i: B_{p, r} \rightarrow \mathbb{D}_{p}(\mu)$ is bounded. Further, the measure $\mu$ is a vanishing $p$-Carleson measure for $B_{p, r}$ if the inclusion operator $i: B_{p, r} \rightarrow$ $\mathbb{D}_{p}(\mu)$ is compact. [1].

The following characterization of $(p, r)$-Carleson measures can be obtained easily from

Theorem 1.3. Take $1<p<\infty$ and $-1<r<\infty$. Let $\mu$ be a positive measure on $\mathbb{D}$. Then the following statements are equivalent:

(1) The measure $\mu$ is a $(p, r)$-Carleson measure for $B_{p, r}$.

(2) There exists a constant $K<\infty$ such that

$$
\mu(S(h, \theta)) \leq K h^{p}
$$

for all $\theta \in[0,2 \pi)$ and $h \in(0,1)$.

(3) There exists a constant $C<\infty$ such that

$$
\int_{\mathbb{D}}\left|\sigma_{a}^{\prime}(z)\right|^{p} d \mu(z) \leq C
$$

for all $a \in \mathbb{D}$.

Using ([6], Lemma 2.1) and ([8], page 163), the following lemma can be proved easily.

Lemma 1.4. Let $\varphi$ be a holomorphic mapping defined on $\mathbb{D}$ such that $\varphi(\mathbb{D}) \subseteq \mathbb{D}$. Take $\psi \in B_{q, r}$ such that $\psi(z) \varphi^{\prime}(z) \in L^{q}\left(\mathbb{D}, d A_{r}\right)$. Then

$$
\int_{\mathbb{D}} g d \mu_{q, r}=\int_{\mathbb{D}}\left|\psi(z) \varphi^{\prime}(z)\right|^{q}(g \circ \varphi)(z)\left(1-|z|^{2}\right)^{r} d A(z)
$$

and

$$
\int_{\mathbb{D}} g d \nu_{q, r}=\int_{\mathbb{D}}\left|\psi^{\prime}(z)\right|^{q}(g \circ \varphi)(z)\left(1-|z|^{2}\right)^{r} d A(z) .
$$

where $g$ is an arbitrary measurable positive function in $\mathbb{D}$. 
We use the following lemma for compactness of the weighted composition operators on Besov-type spaces. The proof of this lemma follows by similar lines as in the case of composition operators on Besov spaces ([12], Lemma 3.8).

Lemma 1.5. Given $1 \leqslant p, q<\infty,-1<r<\infty$, let $\varphi$ be a holomorphic mapping defined on $\mathbb{D}$ with $\varphi(\mathbb{D}) \subseteq \mathbb{D}$ and $\psi \in B_{q, r}$ be such that $W_{\varphi, \psi}: B_{p, r} \rightarrow B_{q, r}$ is bounded. Then $W_{\varphi, \psi}: B_{p, r} \rightarrow B_{q, r}$ is compact (weakly compact) if and only if whenever $\left\{f_{n}\right\}$ is a bounded sequence in $B_{p, r}$ converging to zero uniformly on compact subsets of $\mathbb{D}$, then $\left\|W_{\varphi, \psi}\left(f_{n}\right)\right\|_{B_{q, r}} \rightarrow 0$ (respectivly, $\left\{W_{\varphi, \psi}\left(f_{n}\right)\right\}$ is a weak null sequence in $\left.B_{q, r}\right)$.

Boundededness and compactness of the weighted composition operators on spaces of analytic functions has been studied by many authors. For example we refer to $[2-5,9,10,13]$.

In this article, by using the Carleson measure, we characterize the boundeness and compactness of $W_{\varphi, \psi}$ on Besov-type spaces, in section 2. The Carleson measure characterization of $W_{\varphi, \psi}$ acing on Besov spaces is given in section 3.

\section{Bounded and compact weighted composition operators on Besov-type spaces}

In this section, we characterize the boundeness and compactness of $W_{\varphi, \psi}$ on Besov-type spaces by using Carleson measures.

Theorem 2.1. Take $1<p \leqslant q<\infty$ and $-1<r<\infty$. Let $\varphi \in B_{p, r}$ be such that $\varphi(\mathbb{D}) \subseteq \mathbb{D}, \psi \in A_{r}^{q}$ and the measure $\nu_{q, \psi, r}$ is a vanishing $(q, r)$-Carleson measure for $B_{q, r}$. Then $W_{\varphi, \psi}$ defines a bounded operator from $B_{p, r}$ into $A_{r}^{q}$. Moreover, $W_{\varphi, \psi}: B_{p, r} \rightarrow A_{r}^{q}$ is compact.

Proof. We prove the compactness only. Let $\left\{f_{n}\right\}$ be a bounded sequence in $B_{p, r}$ such that $f_{n} \rightarrow 0$ uniformly on compact subsets of $\mathbb{D}$. Since the measure $\nu_{q, \psi, r}$ is a vanishing $(q, r)$-Carleson measure for $B_{q, r}$, the inclusion map $i: B_{q, r} \rightarrow L^{q}\left(\mathbb{D}, \nu_{q, \psi, r}\right)$ is compact.

Since $B_{p, r} \subset B_{q, r}$, we have $\left\|f_{n}\right\|_{L^{q}\left(\mathbb{D}, \nu_{q, \psi}, r\right.} \rightarrow 0$ as $n \rightarrow \infty$. Therefore, by Lemma 1.4, we have

$$
\begin{aligned}
\left\|W_{\varphi, \psi}\left(f_{n}\right)\right\|_{A_{r}^{q}}^{q} & =\int_{\mathbb{D}}|\psi(z)|^{q}\left|\left(f_{n} \circ \varphi\right)(z)\right|^{q}\left(1-|z|^{2}\right)^{r} d A(z) \\
& =\int_{\mathbb{D}}\left|f_{n}(z)\right|^{q} d \nu_{q, \psi, r}(z) \rightarrow 0, \quad \text { as } \quad n \rightarrow \infty .
\end{aligned}
$$

Thus, $W_{\varphi, \psi}: B_{p, r} \rightarrow A_{r}^{q}$ is compact.

Theorem 2.2. Take $1<p \leqslant q<\infty$ and $-1<r<\infty$. Let $\varphi, \psi \in B_{p, r}$ be such that $\varphi(\mathbb{D}) \subseteq \mathbb{D}$ and the measure $\nu_{q, r}$ is a vanishing $(q, r)$-Carleson measure for $B_{q, r}$. Then $W_{\varphi, \psi}$ is a bounded operator from $B_{p, r}$ into $B_{q, r}$ if and only if $W_{\varphi, \psi \varphi^{\prime}}$ is a bounded operator from $A_{r}^{p}$ into $A_{r}^{q}$.

Proof. Suppose that $W_{\varphi, \psi}: B_{p, r} \rightarrow B_{q, r}$ is bounded. Then there exists a constant $C>0$ such that

$$
\left\|W_{\varphi, \psi}(g)\right\|_{B_{q, r}} \leqslant C\|g\|_{B_{p, r}}
$$

for all $g \in B_{p, r}$. Also, by Theorem 2.1, we can find a constant $M>0$ such that

$$
\left\|W_{\varphi, \psi^{\prime}}(g)\right\|_{A_{r}^{q}} \leqslant M\|g\|_{B_{p, r}}, \quad g \in B_{p, r} .
$$


Take $f \in A_{r}^{p}$ and let the function $g \in B_{p, r}$ be such that $g^{\prime}=f$ and $g(0)=0$. Then,

$$
\begin{aligned}
\left\|W_{\varphi, \psi \varphi^{\prime}}(f)\right\|_{A_{r}^{q}} & =\left\|\psi \varphi^{\prime} f \circ \varphi\right\|_{A_{r}^{q}} \\
& =\left\|\psi \varphi^{\prime} f \circ \varphi+\psi^{\prime} g \circ \varphi-\psi^{\prime} g \circ \varphi\right\|_{A_{r}^{q}} \\
& \leqslant\left\|(\psi g \circ \varphi)^{\prime}\right\|_{A_{r}^{q}}+\left\|\psi^{\prime} g \circ \varphi\right\|_{A_{r}^{q}} \\
& =\|\psi g \circ \varphi\|_{B_{q, r}}+\left\|\psi^{\prime} g \circ \varphi\right\|_{A_{r}^{q}} \\
& \leqslant C\|g\|_{B_{p, r}+M\|g\|_{B_{p, r}}} \\
& =(C+M)\|g\|_{B_{p, r}} \\
& =(C+M)\|f\|_{A_{r}^{p} .}
\end{aligned}
$$

Thus, $W_{\varphi, \psi \varphi^{\prime}}: A_{r}^{p} \rightarrow A_{r}^{q}$ is bounded.

Conversely, suppose $W_{\varphi, \psi \varphi^{\prime}}: A_{r}^{p} \rightarrow A_{r}^{q}$ is bounded. Again, by Theorem 2.1,

$$
W_{\varphi, \psi^{\prime}}: B_{p, r} \rightarrow A_{r}^{q}
$$

is bounded. Take $f \in B_{p, r}$ such that $f(0)=0$. Then, we have

$$
\begin{aligned}
\left\|W_{\varphi, \psi}(f)\right\|_{B_{q, r}} & =\left\|(\psi f \circ \varphi)^{\prime}\right\|_{A_{r}^{q}} \\
& =\left\|\psi \varphi^{\prime} f^{\prime} \circ \varphi+\psi^{\prime} f \circ \varphi\right\|_{A_{r}^{q}} \\
& \leqslant\left\|W_{\varphi, \psi \varphi^{\prime}}\left(f^{\prime}\right)\right\|_{A_{r}^{q}}+\left\|W_{\varphi, \psi^{\prime}}(f)\right\|_{A_{r}^{q}}<+\infty .
\end{aligned}
$$

The theorem is proved.

Theorem 2.3. Take $1<p \leqslant q<\infty$ and $-1<r<\infty$. Let $\varphi, \psi \in B_{p, r}$ be such that $\varphi(\mathbb{D}) \subseteq \mathbb{D}$ and the measure $\nu_{q, r}$ is a vanishing $(q, r)$-Carleson measure for $B_{q, r}$. Then $W_{\varphi, \psi}$ is a compact operator from $B_{p, r}$ into $B_{q, r}$ if and only if $W_{\varphi, \psi \varphi^{\prime}}$ is a compact operator from $A_{r}^{p}$ into $A_{r}^{q}$.

Proof. Suppose that $W_{\varphi, \psi}: B_{p, r} \rightarrow B_{q, r}$ is compact. Let $\left\{f_{n}\right\}$ be a bounded sequence in $A_{r}^{p}$ such that $f_{n} \rightarrow 0$ uniformly on compact subsets of $\mathbb{D}$. For each $n$, there exists a function $g_{n} \in B_{p, r}$ such that $g_{n}^{\prime}=f_{n}$ and $g_{n}(0)=0$. The sequence $\left\{g_{n}\right\}$ also converges to zero uniformly on compact subsets of $\mathbb{D}$ as $n \rightarrow \infty$. Further, since $W_{\varphi, \psi}: B_{p, r} \rightarrow B_{q, r}$ is compact, so $\left\|W_{\varphi, \psi}\left(g_{n}\right)\right\|_{B_{q, r}} \rightarrow 0$ as $n \rightarrow \infty$. Again, by Theorem 2.1, $W_{\varphi, \psi^{\prime}}: B_{p, r} \rightarrow A_{r}^{q}$ is compact, so $\left\|W_{\varphi, \psi^{\prime}}\left(g_{n}\right)\right\|_{A_{r}^{q}}$ also converges to zero as $n \rightarrow \infty$. We have

$$
\begin{aligned}
\left\|W_{\varphi, \psi \varphi^{\prime}}\left(f_{n}\right)\right\|_{A_{r}^{q}} & =\left\|\psi \varphi^{\prime} f_{n} \circ \varphi\right\|_{A_{r}^{q}} \\
& \leqslant\left\|\psi \varphi^{\prime} f_{n} \circ \varphi+\psi^{\prime} g_{n} \circ \varphi\right\|_{A_{r}^{q}}+\left\|\psi^{\prime} g_{n} \circ \varphi\right\|_{A_{r}^{q}} \\
& =\left\|\left(\psi g_{n} \circ \varphi\right)^{\prime}\right\|_{A_{r}^{q}}+\left\|W_{\varphi, \psi^{\prime}}\left(g_{n}\right)\right\|_{A_{r}^{q}} \\
& =\left\|W_{\varphi, \psi}\left(g_{n}\right)\right\|_{B_{q, r}}+\left\|W_{\varphi, \psi^{\prime}}\left(g_{n}\right)\right\|_{A_{r}^{q}} \rightarrow 0, \quad \text { as } \quad n \rightarrow \infty .
\end{aligned}
$$

Therefore, $W_{\varphi, \psi \varphi^{\prime}}: A_{r}^{p} \rightarrow A_{r}^{q}$ is compact.

Conversely, suppose $W_{\varphi, \psi \varphi^{\prime}}: A_{r}^{p} \rightarrow A_{r}^{q}$ is compact. Again, by Theorem 2.1, $W_{\varphi, \psi^{\prime}}: B_{p, r} \rightarrow$ $A_{r}^{q}$ is compact. Let $\left\{g_{n}\right\}$ be the same sequence as in the direct part. Then,

$$
\begin{aligned}
\left\|W_{\varphi, \psi}\left(g_{n}\right)\right\|_{B_{q, r}}= & \left\|\left(\psi g_{n} \circ \varphi\right)^{\prime}\right\|_{A_{r}^{q}} \\
= & \left\|\psi \varphi^{\prime} g_{n}^{\prime} \circ \varphi+\psi^{\prime} g_{n} \circ \varphi\right\|_{A_{r}^{q}} \\
& \left\|W_{\varphi, \psi \varphi^{\prime}}\left(f_{n}\right)\right\|_{A_{r}^{q}+\| W_{\varphi, \psi^{\prime}}}\left(g_{n}\right) \|_{A_{r}^{q}} \rightarrow 0, \quad \text { as } \quad n \rightarrow \infty .
\end{aligned}
$$

Thus, $W_{\varphi, \psi}: B_{p, r} \rightarrow B_{q, r}$ is compact.

Theorem 2.4. Take $1<p<\infty$ and $-1<r<\infty$. Let $\varphi, \psi \in B_{p, r}$ be such that $\varphi(\mathbb{D}) \subseteq \mathbb{D}$ and the measure $\nu_{p, r}$ is a vanishing $(p, r)$-Carleson measure for $B_{p, r}$. Then $W_{\varphi, \psi}$ is a bounded (compact) operator from $B_{p, r}$ into $B_{p, r}$ if and only if $\mu_{p, r}$ is a bounded (vanishing) $(p, r)$-Carleson measure for $B_{p, r}$.

Proof. We only prove the boundedness. Suppose first that $W_{\varphi, \psi}: B_{p, r} \rightarrow B_{p, r}$ is bounded. Then by Theorem $2.2, W_{\varphi, \psi \varphi^{\prime}}$ is a bounded operator on $A_{r}^{p}$. Let $f \in B_{p, r}$ be such that 
$f(0)=0$. Then, by using Lemma 1.4 , we have

$$
\begin{aligned}
\left\|W_{\varphi, \psi \varphi^{\prime}}\left(f^{\prime}\right)\right\|_{A_{r}^{p}}^{p} & =\int_{\mathbb{D}}\left|\psi(z) \varphi^{\prime}(z)\right|^{p}\left|f^{\prime}(\varphi(z))\right|^{p}\left(1-|z|^{2}\right)^{r} d A(z) \\
& =\int_{\mathbb{D}}\left|f^{\prime}(w)\right|^{p} d \mu_{p, r}(w) .
\end{aligned}
$$

Since $W_{\varphi, \psi \varphi^{\prime}}$ is bounded on $A_{r}^{p}$, therefore we can find a constant $C>0$ such that

$$
\left\|W_{\varphi, \psi \varphi^{\prime}}\left(f^{\prime}\right)\right\|_{A_{r}^{p}}^{p} \leqslant C\left\|f^{\prime}\right\|_{A_{r}^{p}}^{p} .
$$

Hence,

$$
\int_{\mathbb{D}}\left|f^{\prime}(w)\right|^{p} d \mu_{p, r}(w) \leqslant C\|f\|_{B_{p, r}}^{p} .
$$

That is, the inclusion operator $i: B_{p, r} \rightarrow \mathbb{D}_{p, r}(\mu)$ is bounded. Thus the measure $\mu_{p, r}$ is a bounded $(p, r)$-Carleson measure for $B_{p, r}$.

Conversely, suppose that $\mu_{p, r}$ is a bounded $(p, r)$-Carleson measure for $B_{p, r}$. We want to show that $W_{\varphi, \psi}: B_{p, r} \rightarrow B_{p, r}$ is bounded. We have

$$
\left(\psi(f \circ \varphi)^{\prime}\right)=\psi \varphi^{\prime}\left(f^{\prime} \circ \varphi\right)+\psi^{\prime}(f \circ \varphi) .
$$

Take $f \in B_{p, r}$. So by Lemma 1.4,

$$
\int_{\mathbb{D}}\left|\psi(z) \varphi^{\prime}(z)\right|^{p}\left|f^{\prime}(\varphi(z))\right|^{p}\left(1-|z|^{2}\right)^{r} d A(z)=\int_{\mathbb{D}}\left|f^{\prime}(w)\right|^{p} d \mu_{p, r}(w)<+\infty .
$$

Also, by using Theorem 2.1, we get

$$
\int_{\mathbb{D}}\left|\psi^{\prime}(z)\right|^{p}|f(\varphi(z))|^{p}\left(1-|z|^{2}\right)^{r} d A(z)=\left\|W_{\varphi, \psi^{\prime}}(f)\right\|_{A_{r}^{p}}^{p}<+\infty .
$$

By using (2.2), (2.3) and (2.4), $W_{\varphi, \psi}: B_{p, r} \rightarrow B_{p, r}$ is bounded.

Compactness of $W_{\varphi, \psi}$ can be proved by using the Theorems 2.1 and 2.3 , which we omit its proof.

\section{Carleson measure characterization of the weighted composition oper- ators on Besov spaces}

In this section, we give a Carleson measure charecterization of $W_{\varphi, \psi}$ on Besov space.

Let $1<p, q<\infty, \varphi$ be a holomorphic mapping defined on $\mathbb{D}$ such that $\varphi(\mathbb{D}) \subseteq \mathbb{D}$ and $\psi \in B_{q}$ be such that $\psi(z) \varphi^{\prime}(z)\left(1-|z|^{2}\right) \in L^{q}(\mathbb{D}, d \lambda)$ (where $d \lambda(z)=\left(1-|z|^{2}\right)^{-2} d A(z)$ is the Mobius invariant measure on $\mathbb{D}$ ). For $f \in B_{p}$ there exists a constant $C_{q}$ such that

$$
\begin{aligned}
\left\|W_{\varphi, \psi}(f)\right\|_{B_{q}}^{q}= & \int_{\mathbb{D}}\left|\left(\psi C_{\varphi} f\right)^{\prime}(z)\right|^{q}\left(1-|z|^{2}\right)^{q-2} d A(z) \\
\leqslant & C_{q} \int_{\mathbb{D}}\left|\psi^{\prime}(z)\right|^{q}\left|\left(C_{\varphi} f\right)(z)\right|^{q}\left(1-|z|^{2}\right)^{q-2} d A(z) \\
& +C_{q} \int_{\mathbb{D}}|\psi(z)|^{q}\left|\varphi^{\prime}(z)\right|^{q}\left|f^{\prime}(\varphi(z))\right|^{q}\left(1-|z|^{2}\right)^{q-2} d A(z) .
\end{aligned}
$$

By using Lemma 1.4, we have

$$
\left\|W_{\varphi, \psi}(f)\right\|_{B_{q}}^{q} \leqslant C_{q} \int_{\mathbb{D}}|f(w)|^{q} d \nu_{q}(w)+C_{q} \int_{\mathbb{D}}\left|f^{\prime}(w)\right|^{q} d \mu_{q}(w) .
$$

Since $W_{\varphi, \psi}: B_{p} \rightarrow B_{q}$ is a bounded operator if and only if there exists a positive constant $C$ such that

$$
\left\|W_{\varphi, \psi}(f)\right\|_{B_{q}}^{q} \leq C\|f\|_{B_{p}}^{q},
$$

so, the following theorem holds. 
Theorem 3.1. Let $1<p<\infty, \varphi$ be a holomorphic mapping defined on $\mathbb{D}$ such that $\varphi(\mathbb{D}) \subseteq \mathbb{D}$ and $\psi \in B_{p}$ be such that $\psi(z) \varphi^{\prime}(z)\left(1-|z|^{2}\right) \in L^{p}(\mathbb{D}, d \lambda)$. If the measure $\mu_{p}$ is a $p$ Carleson measure and $\nu_{p}$ is a vanishing $p$-Carleson measure for $B_{p}$, then $W_{\varphi, \psi}: B_{p} \rightarrow B_{p}$ is a bounded operator.

Proof. Suppose that $\mu_{p}$ is a $p$-Carleson measure for $B_{p}$. By Definition 1.2, there exisits a constant $C_{1}$ such that

$$
\int_{D}\left|f^{\prime}(w)\right|^{p} d \mu_{p}(w) \leq C_{1}\|f\|_{B_{p}}^{p} .
$$

Let $\nu_{p}$ is a vanishing $p$-Carleson measure for $B_{p}$. By using Theorem 2.1 for $r=p-2$, there exisits a constant $C_{2}$ such that

$$
\begin{aligned}
\left\|W_{\varphi, \psi^{\prime}}(f)\right\|_{A^{p}}^{p} & =\int_{D}\left|\psi^{\prime}(z)\right|^{p}|f \circ \varphi|^{p}\left(1-|z|^{2}\right)^{p-2} d A z \\
& =\int_{D}|f(w)|^{p} d \nu_{p}(w) \\
& \leq C_{2}\|f\|_{B_{p}}^{p} .
\end{aligned}
$$

By using (3.2) and (3.3), from (3.1) the theorem is proved.

Theorem 3.2. Suppose $1<p \leqslant q<\infty$ and $\varphi$ is a holomorphic mapping defined on $\mathbb{D}$. Let $\varphi(\mathbb{D}) \subseteq \mathbb{D}$ and $\psi \in B_{q}$ be such that $\psi(z) \varphi^{\prime}(z)\left(1-|z|^{2}\right) \in L^{q}(\mathbb{D}, d \lambda)$. If the measures $\nu_{q}$ and $\mu_{q}$ are vanishing $q$-Carleson measures for $B_{q}$, then $W_{\varphi, \psi}: B_{p} \rightarrow B_{q}$ is a compact operator.

Proof. Let $\left\{f_{n}\right\}$ be a bounded sequence in $B_{p}$ such that $f_{n} \rightarrow 0$ uniformly on compact subsets of $\mathbb{D}$. Then the mean value property for the holomorphic function yields

$$
f_{n}^{\prime}(w)=\frac{4}{\pi(1-|w|)^{2}} \int_{|w-z|<\frac{1-|w|}{2}} f_{n}^{\prime}(z) d A(z) .
$$

Therefore by Jensen's inequality,

$$
\left|f_{n}^{\prime}(w)\right|^{q} \leq \frac{4}{\pi(1-|w|)^{2}} \int_{|w-z|<\frac{1-|w|}{2}}\left|f_{n}^{\prime}(z)\right|^{q} d A(z) .
$$

Since the measure $\nu_{q}$ is a vanishing $q$-Carleson measure for $B_{q}$, by using Theorem 2.1(relation (2.1)), we have

$$
\int_{D}\left|f_{n}(w)\right|^{q} d \nu_{q}(w) \rightarrow 0, \text { as } n \rightarrow \infty
$$

By using (3.1), (3.5), (3.6) and Fubini's Theorem,

$$
\begin{aligned}
\left\|W_{\varphi, \psi}\left(f_{n}\right)\right\|_{B_{q}}^{q} & \leq C_{q} \int_{D}\left|f_{n}(w)\right|^{q} d \nu_{q}(w)+C_{q} \int_{D}\left|f_{n}^{\prime}(w)\right|^{q} d \mu_{q}(w) \\
& \leq C_{q} \int_{D} \frac{4}{\pi(1-|w|)^{2}}\left(\int_{|w-z|<\frac{1-|w|}{2}}\left|f_{n}^{\prime}(z)\right|^{q} d A(z)\right) d \mu_{q}(w) \\
& \leq C_{q} \frac{4}{\pi} \int_{D}\left|f_{n}^{\prime}(z)\right|^{q}\left(\int_{D} \frac{1}{(1-|w|)^{2}} \chi_{\left\{z:|w-z|<\frac{1-|w|}{2}\right\}}(z) d \mu_{q}(w)\right) d A(z) .
\end{aligned}
$$

Note that if $|w-z|<\frac{1-|w|}{2}$, then $w \in S(2(1-|z|), \theta)$, where $z=|z| e^{i \theta}$, since

$$
\left|w-e^{i \theta}\right| \leq|z-w|+\left|e^{i \theta}-z\right|<\frac{1-|w|}{2}+\left|\frac{z}{|z|}-z\right|<2(1-|z|) .
$$

Moreover, if $|w-z|<\frac{1-|w|}{2}$ then

$$
\frac{1}{(1-|w|)^{2}} \leq \text { const. } \frac{1}{(1-|z|)^{2}} \text {. }
$$


Hence,

$$
\begin{aligned}
\left\|W_{\varphi, \psi}\left(f_{n}\right)\right\|_{B_{q}}^{q} & \leq \text { const. } \int_{D} \frac{\left|f_{n}^{\prime}(z)\right|^{q}}{(1-|z|)^{2}}\left(\int_{S(2(1-|z|), \theta)} d \mu_{q}(w)\right) d A(z) \\
& =\text { const. }\left(\int_{|z|>1-\frac{\delta}{2}}+\int_{|z| \leq 1-\frac{\delta}{2}} \frac{\left|f_{n}^{\prime}(z)\right|^{q}}{(1-|z|)^{2}}\left(\int_{S(2(1-|z|), \theta)} d \mu_{q}(w)\right) d A(z)\right) \\
& =I+I I
\end{aligned}
$$

for any $0<\delta<1$.

Fix $\epsilon>0$ and let $\delta>0$ be such that for any $\theta \in[0,2 \pi]$ and any $h<\delta$,

$$
\mu_{q}(S(h, \theta))<\epsilon h^{q},
$$

and so

$$
\int_{S(h, \theta)} d \mu_{q}<\epsilon h^{q}
$$

By (3.8),

$$
\begin{aligned}
I & \leq \text { const. } 2^{q} \epsilon \int_{|z|>1-\frac{\delta}{2}} \frac{\left|f_{n}^{\prime}(z)\right|^{q}}{\left(1-|z|^{2}\right)^{2}}\left(1-|z|^{2}\right)^{q} d A(z) \\
& \leq \text { const. } \epsilon\left\|f_{n}\right\|_{B_{q}}^{q}<\text { const. } \epsilon .
\end{aligned}
$$

For $n$ large enough, since $f_{n}^{\prime} \rightarrow 0$ uniformly on compact sets, we have

$$
I I \leq \text { const. } \int_{|z| \leq 1-\frac{\delta}{2}}\left|f_{n}^{\prime}(z)\right|^{q}\left(\int_{D} d \mu_{q}\right) d A(z)<\text { const. } \epsilon .
$$

Therefore, from (3.7), (3.9) and (3.10) we obtain

$$
\left\|W_{\varphi, \psi}\left(f_{n}\right)\right\|_{B_{q}}^{q}<\text { const. } \epsilon .
$$

Thus $\left\|W_{\varphi, \psi}\left(f_{n}\right)\right\|_{B_{q}}^{q} \rightarrow 0$ as $n \rightarrow \infty$, and from Lemma $1.5, W_{\varphi, \psi}$ is compact.

\section{References}

[1] J. Arazy, S.D. Fisher and J. Peetre, Mobius invariant function spaces, J. Reine Angew. Math. 363, 110-145, 1985.

[2] D. Blasi and J. Pav, A Characterization of Besov type spaces and applications to Hankel-type operators, Michigan Math. J. 56, 401-418, 2008.

[3] F. Colonna and S. Li, Weighted Composition Operators from the Besov spaces into the Bloch space, Bull. Malays. Math. Sci. Soc. 36, 1027-1039, 2013.

[4] F. Colonna and M. Tjani, Weighted Composition Operators from the Besov spaces into the weighted type space $H_{\mu}^{\infty}$, J. Math. Anal. Appl. 402, 594-611, 2013.

[5] F. Colonna and M. Tjani, Weighted Composition Operators from the analytic Besov spaces to BMOA, Oper. Theory Adv. Appl. 236, 133-157, 2014.

[6] M.D. Contreras and A.G. Hernandez-Diaz, Weighted composition operators on Hardy spaces, J. Math. Anal. Appl. 263, 224-233, 2001.

[7] C.C. Cowen and B.D. MacCluer, Composition operators on spaces of analytic functions, CRC Press, Boca Raton, New York, 1995.

[8] P.R. Halmos, Measure Theory, Grad. Texts in Math., 18, Springer-Verlag, New York, 1974.

[9] M. Hassanlou, J. Laitila and H. Vaezi, Weighted composition operators between weak spaces of vector-valued analytic functions, Analysis, 37, 39-45, 2017.

[10] M. Hassanlou, H. Vaezi and M. Wang, Weighted composition operators on weak vectorvalued Bergman spaces and Hardy spaces, Banach J. Math. Anal. 9 (2), 35-43, 2015.

[11] J.H. Shapiro, Composition operators and classical function theory, Berlin, Germany, Springer- Verlag, 1993. 
[12] M. Tjani, Compact composition operators on Besov space, Trans. Amer. Math. Soc. 355 (11), 4683-4698, 2003.

[13] W. Yang, Weighted composition operators from Bloch-type spaces to weighted-type spaces, Ars. Combin. 92, 415-423, 2009. 